\title{
bDMARD-Therapie der Rheumatoiden Arthritis in Österreich
}

Eine im BMC Musculoskeletal Disorders erschienene Arbeit untersuchte die Validität der im Biologika-Register für entzündliche rheumatische Erkrankungen (BioReg) gesammelten Daten bezüglich der gegenwärtigen Praxis der bDMARD-Therapie der rheumatoiden Arthritis.

Das Biologika-Register für entzündliche rheumatische Erkrankungen (BioReg) begann 2010 mit der Datenerfassung. Hauptziel des Registers ist, Daten über die Therapiesicherheit zu erfassen, daneben aber auch Effektivität der Therapie und Therapieverläufe bzw. -änderungen zu dokumentieren, sowie sozio-ökonomische Daten zu sammeln. Mittlerweile beteiligen sich 25 österreichische Zentren aus allen neun Bundesländern an der Datenerfassung (zwölf Rheumatologen in Privatordinationen und dreizehn spezialisierte Ambulanzen in öffentlichen und privaten Krankenanstalten).

Ziel der Untersuchung war es, die Validität der im BioReg gesammelten Daten zu überprüfen und etwaige $A b$ weichungen im Hinblick auf die Krankheitsaktivität nach einem Jahr Therapie bei zwei Gruppen von Patienten mit Rheumatoider Arthritis (RA) aufzudecken: Verglichen wurden Patientinnen und Patienten, die zum Zeitpunkt ihrer Aufnahme in das Register bereits unter einer Behandlung mit Biologika (biological disease-modifying antirheumatic drugs, bDMARDs) standen (EST) mit jenen PatientInnen, die zum Zeitpunkt ihrer Aufnahme in das Register die Behandlung mit bDMARDs begannen (NEW).

Die globale Krankheitsaktivität, Remissionsraten, begleitende konventionelle synthetische DMARDs (conventional synthetic (cs) DMARDs), begleitende Glukokortikoidtherapie und Nebenwirkungen wurden zu Beginn und nach einem Jahr evaluiert. Der Ausgangshypothese zufolge sollte die Krankheitsaktivität der EST-Gruppe mit jener der NEW-Gruppe nach einem Jahr vergleichbar sein.

Insgesamt sind derzeit Daten von 970 RA-Patienten im Register enthalten; 346 RA-Patienten (284 EST, 62 $\mathrm{NEW}$ ), von denen ein vollständiger Datensatz, sowohl zu Baseline als auch beim 1-Jahres-Follow-Up, vorhanden war, wurden in die Analyse inkludiert.

Nach einem Jahr ließen sich mittels DAS28 und RADAl-5 keine Unterschiede in der Krankheitsaktivität feststellen und hinsichtlich der Remissionsraten zeigten sich lediglich kleine Unterschiede je nach Anwendung von DAS28, RADAI-5 oder der Boolean Definition von RA-Remission. 64 Nebenwirkungen (AE) wurden bei 56 (20\%) der EST-Patienten und 20 AEs bei 19 (31\%) der NEW-Patienten erfasst. Malignitäten traten bei 4 Patienten auf. Nach einem Jahr befanden sich $48 \%$ der EST-Patienten, aber nur $16 \%$ der NEW-Patienten, auf einer bDMARD-Monotherapie.

Die Autoren ziehen den Schluss, dass die im BioReg erhobenen Daten als valide angenommen werden können. Nach einem Jahr Dokumentation im Register und bDMARD-Therapie erreichten alle Patienten (EST und NEW) ein vergleichbares Niveau der Krankheitsaktivität. Nebenwirkungen traten häufiger während der frühen Phase der bDMARD-Behandlung auf, wie auch schon aus Registern anderer europäischer Länder bekannt war.

Die Plausibilität der Erhebung wurde durch einen Vergleich mit dem deutschen Register zur Langzeitbeobachtung der Biologikatherapie bei erwachsenen Patienten mit RA (RABBIT) zusätzlich erhärtet. Der einzig ins Auge springende
Unterschied besteht in der hohen Rate an schwerwiegenden Nebenwirkungen in Form von Infektionen bei Patienten derEST-Gruppe. Dieser Unterschied kann allerdings zumindest teilweise durch die höhere Frequenz von Krankenhausaufnahmen in Österreich erklärt werden.

Österreichs Rheumatologen initiieren - im Vergleich zu europäischen Kollegen - eine bDMARD-Therapie bereits bei Patienten mit hoch moderater Krankheitsaktivität (also im Schnitt bei weniger aktiven Patienten), was offenbar zu höheren Remissionsraten führt. Zudem scheint es zum österreichischen BehandlungsStandard zu gehören, die Behandlung mit csDMARDs und Glukokortikoiden im Falle des günstigen Therapieverlaufes zu reduzieren bzw. zu beenden, während die betroffenen Patienten weiter ihre bDMARDs erhalten, um den therapeutischen Erfolg zu erhalten.

rheuma plus $2017 \cdot 16: 11$

DOI 10.1007/s12688-017-0106-6

Online publiziert: 2. Februar 2017

(c) Springer-Verlag Wien 2017

Quelle: Rintelen B. et al.: Validity of data collected in BIOREG, the Austrian register for biological treatment in rheumatology: current practice of bDMARD therapy in rheumatoid arthritis in Austria, BMC Musculoskeletal Disorders (2016) 17:358, doi $10.1186 / 512891-016-$ $1207-4$
Hier steht eine Anzeige.

\section{Springer}

